\title{
The challenge of hyperon polarization
}

\author{
S. M. Troshin, N. E. Tyurin \\ Institute for High Energy Physics, \\ Protvino, Moscow Region, 142280, Russia
}

\begin{abstract}
We provide a brief topical outline of the persisting problem of hyperon polarization and consider some future experimental prospects. Predictions which deserve experimental verification are proposed.
\end{abstract}




\section{Inroduction}

In the recent years a number of significant and unexpected spin effects were discovered. They demonstrated that the spin degrees of freedom will be important even at $\mathrm{TeV}$ energy scale. Currently spin experiments are conducted at almost all existing accelerators and are planned for those under construction. The experimental data on the spin observables represent a rather small fraction of the data on particle interactions - most of the data are related to spin-averaged ones. Despite that, these data prove to be very important for understanding of particle interactions. They provide information on the spin dependence of the interactions, which in some sense is more fundamental than the dynamics studied using spinaveraged observables. These data clearly indicate that high energy interaction dynamics depends significantly on the spin degrees of freedom. Last year with the start of the RHIC spin program [1], spin studies are moving again to the forefront of high energy physics.

The spin experiments aim at:

- the study of the spin structure of the nucleon, i.e., how the proton's spin state can be obtained from a superposition of Fock states with different numbers of constituents with nonzero spin;

- the study of how the dynamics of constituent interactions depends on the spin degrees of freedom;

- to understand chiral symmetry breaking and helicity non-conservation on the quark and hadron levels;

- study the overall nucleon structure and long range dynamics.

Those issues are closely interrelated at the hadron level and the results of the experimental measurements can be interpreted in terms of hadron spin structure convoluted with the constituent interaction dynamics.

In deep-inelastic scattering data analysis in the framework of perturbative QCD provides information on the longitudinal spin parton densities $\Delta q(x)$. The study of the transverse spin parton densities $\delta q(x)$ has recently become also an equally actual problem. However, in the usual deep-inelastic scattering the transverse spin contribute only as higher-twist contributions. The transverse spin densities can be studied in the Drell-Yan processes or in specific semi-inclusive DIS reactions [1]].

In soft hadronic interactions significant single-spin effects could be expected since the helicity conservation does not work for interactions at large distances, once the chiral $S U(3)_{L} \times S U(3)_{R}$ symmetry of the QCD Lagrangian is spontaneously broken. However, the spin asymmetry $A_{N}$ at low transverse momentum 
found to be small and decreases with energy. In contrast, the spin asymmetry increases at high transverse momentum in elastic scattering, where we should expect a decreasing behaviour based on the helicity conservation due to the chiral invariance of perturbative QCD. More theoretical work and experimental data will be needed to understand the dynamics of these unexpected single-spin effects observed in elastic scattering and inclusive hyperon and meson production. In the following we consider these issues concentrating on the particular problem of hyperon polarization.

\section{Experimental status}

In 1976 it was discovered that in the reaction $p p \rightarrow \Lambda X$ highly polarized $\Lambda_{-}$ hyperons were produced, and their polarization rises with $x_{F}$ and $p_{\perp}$ [2]. Nowadays there is a rather extensive experimental set of data on the hyperon polarization in inclusive reactions [3, 团]. The fact that polarization of the $\Lambda$ enters in the formula for the proton angular distribution in the $\Lambda \rightarrow p \pi^{-}$decay, which does not conserve parity, allows to perform its measurement. The polarization of $\Lambda$ is obtained from the angular-distribution analysis of the decay products. After the $\Lambda$-hyperon polarization discovery, the polarization of other hyperons was measured and they also appeared to be polarized. The experimental studies revealed the following regularities [3, 勾]:

- In proton-proton interactions, the polarization of inclusively produced $\Lambda$ hyperons is negative, its direction is opposite to the vector $\vec{p} \times \vec{p}_{\Lambda}$, where $\vec{p}$ is the incident particle momentum. The polarization rises with transverse momentum of $\Lambda$-particle. For the range of transverse momentum $p_{\perp}>0.8$ $\mathrm{GeV} / c$ the dependence of $P_{\Lambda}$ on the transverse momentum becomes weak. In this region of $p_{\perp}$, the polarization of $\Lambda$ grows linearly with $x_{F}$. The $\Lambda$ hyperon polarization has been measured up to rather high values of $p_{\perp} \simeq$ $3.5 \mathrm{GeV} / c$. However, even at the maximum values of $p_{\perp}$ no tendency for a decreasing polarization was observed. It is interesting that spin asymmetry $A_{N}$ and spin transfer parameter $D_{N N}$ have shown similar $p_{\perp}$-dependence in $\Lambda$-production at FNAL [ $\boxminus$.

- The $\Lambda$-hyperon polarization is energy independent in a wide range of beam energy $p=12-2000 \mathrm{GeV} / \mathrm{c}$.

- In proton-proton interactions, hyperon production reactions reveal the following relations between the hyperon polarizations:

$$
P_{\Sigma^{0}} \simeq P_{\Sigma^{-}} \simeq P_{\Sigma^{+}} \simeq-P_{\Lambda} \simeq-P_{\Xi^{0}} \simeq-P_{\Xi^{-}},
$$




$$
P_{\bar{\Lambda}} \simeq P_{p} \simeq P_{\Omega^{-}} \simeq 0, \quad P_{\Lambda}(p \rightarrow \Lambda) \simeq P_{\bar{\Lambda}}(\bar{p} \rightarrow \bar{\Lambda}) .
$$

- For the reactions involving mesons the following relations are observed:

$$
P_{\Lambda}\left(K^{-} \rightarrow \Lambda\right)>0, \quad\left|P_{\Lambda}\left(K^{-} \rightarrow \Lambda\right)\right| \gg\left|P_{\Lambda}(p \rightarrow \Lambda)\right|
$$

and

$$
\left|P_{\bar{\Lambda}}\left(K^{+} \rightarrow \bar{\Lambda}\right)\right| \gg\left|P_{\bar{\Lambda}}(\bar{p} \rightarrow \bar{\Lambda})\right| .
$$

The polarization of $\Xi^{-}$-hyperons produced by $800-\mathrm{GeV}$ protons has also been measured at FNAL. Contrary to the regularities observed in the $\Lambda$-production, energy dependence of $P_{\Xi^{-}}$has been observed for the first time. The quantity $P_{\Xi^{-}}$ also does not show the $x_{F}$-dependence as observed in $\Lambda$-polarization data.

To determine the polarization of directly produced hyperons one has to measure the hyperon polarization in exclusive reactions. To this end, the $\Lambda$-hyperon polarization has been studied [5] in the exclusive diffractive process

$$
p+p \rightarrow p+\left[\Lambda^{0} K^{+}\right] .
$$

Results of the measurements demonstrated that $\Lambda$-hyperon polarization increases linearly with $p_{\perp}$ at $\sqrt{s} \simeq 30 \mathrm{GeV}$ and gets a very large value, $P_{\Lambda} \simeq 80 \%$, at $p_{\perp} \simeq 1 \mathrm{GeV} / \mathrm{c}$.

Therefore, since the $\Lambda$ polarization does not show a decrease at large $p_{\perp}$ values, the data on hyperon polarization provide a direct evidence for the $s-$ channel helicity nonconservation in hadron collisions in a wide energy and $p_{\perp}$ range.

\section{Theoretical status}

The theoretical status of hyperon polarization was described in many review papers (see e. g. [6]). Hyperon polarization even the most simple case - the polarization of $\Lambda$ 's - is not understood in $\mathrm{pQCD}$ and it seems that in the future it could become an even more serious problem than the nucleon spin problem. Those problems are likely related. One could attempt to connect the spin structure studied in deep-inelastic scattering with the polarization of $\Lambda$ 's observed in hadron production [7]. As is widely known now, only a part (less than one third) of the proton spin is due to spins of the quarks. Studies of spin effects in inclusive processes yield information on the contribution of the spin and orbital angular momenta of quarks and gluons into the hadron helicity:

$$
1 / 2=1 / 2 \Delta \Sigma+L_{q}+\Gamma+L_{g},
$$


In the sum above first two terms are the contributions of spin and orbital momentum of the quarks and last two terms are the corresponding quantities related to the gluons, i.e. all these terms have a clear physical interpretation; however besides the first one, these are gauge and frame dependent.

Experimental results on the nucleon structure can be interpreted in an effective QCD approach ascribing a substantial part of the hadron spin to orbital angular momentum of the quark matter. It is natural to assume that this orbital angular momentum could be the origin of the asymmetries in hadron production. It is also evident from the recent deep-inelastic scattering data that strange quarks play essential role in the proton structure and in its spin balance. Polarization effects in hyperon production are complimentary to these results and demonstrate [3] also that strange quarks acquire polarization in the course of the hadron interactions. The explicit formula for the asymmetry in terms of the helicity amplitudes has the following form:

$$
P, A_{N}=2 \frac{\sum_{X, \lambda_{X}, \lambda_{2}} \int d \Gamma_{X} \operatorname{Im}\left[F_{\lambda_{X} ; \lambda_{2}} F_{\lambda_{X} ;-, \lambda_{2}}^{*}\right]}{\sum_{X, \lambda_{X} ; \lambda_{1} \lambda_{2}} \int d \Gamma_{X}\left|F_{\lambda_{X} ; \lambda_{1}, \lambda_{2}}\right|^{2}}
$$

and shows that non-zero single spin asymmetries require the presence of helicity flip and nonflip amplitudes and a phase difference between those amplitudes. A straightforward application of perturbative QCD using collinear factorization scheme, where e. g.

$$
A_{N} d \sigma \sim \sum_{a b \rightarrow c d} \int d \xi_{A} d \xi_{B} \frac{d z}{z} \delta f_{a / A}\left(\xi_{A}\right) f_{b / B}\left(\xi_{B}\right) \hat{a}_{N} d \hat{\sigma}_{a b \rightarrow c d} D_{C / c}(z)
$$

encounters serious difficulties in trying to explain the measured polarization results. This is due to the chiral invariance and vector nature of QCD Lagrangian which provide important consequences for spin observables and therefore allows for direct and unambiguous experimental test of perturbative QCD. Indeed, the QCD Lagrangian is invariant under transformation of $S U(3)_{L} \times S U(3)_{R}$ group $\left(N_{f}=3\right)$ and therefore QCD interactions are the same for left and right quarks:

$$
\bar{\psi} \gamma_{\mu} \psi A^{\mu}=\bar{\psi}_{L} \gamma_{\mu} \psi_{L} A^{\mu}+\bar{\psi}_{R} \gamma_{\mu} \psi_{R} A^{\mu}
$$

Left-handed (right-handed) massless particles will always stay left handed (righthanded). For massless quarks chirality and helicity coincide. If the quarks have a non-zero mass chirality and helicity are only approximately equal at high energies, namely:

$$
\psi_{1 / 2}=\psi_{R}+O\left(\frac{m}{\sqrt{\hat{s}}}\right) \psi_{L}, \quad \psi_{-1 / 2}=\psi_{L}+O\left(\frac{m}{\sqrt{\hat{s}}}\right) \psi_{R}
$$


where $\pm 1 / 2$ are the quark helicities. Any quark line entering a Feynman diagram corresponding the QCD Lagrangian emerges from it with unchanged helicity. Quark helicity conservation is the most characteristic feature of this theory. To get quark polarization values $\hat{P}_{q} \neq 0$ it is necessary that helicity flip amplitude is a non-zero and that phases of helicity flip $F_{f}$ and non-flip $F_{n f}$ amplitudes are different, since

$$
\hat{a}_{N}, \hat{P}_{q} \propto \operatorname{Im}\left(F_{n f} F_{f}^{*}\right)
$$

In QCD the quark helicity flip amplitude is of order of $m / \sqrt{\hat{s}}$. In the Born approximation the amplitudes are real and therefore one needs to consider the loop diagrams , i.e. the quark helicity flip amplitude will be proportional to

$$
F_{f}^{q} \propto \frac{\alpha_{s} m_{q}}{\sqrt{\hat{s}}} F_{n f}^{q}
$$

and consequently the quark polarization is vanishingly small in hard interactions [8]:

$$
\hat{a}_{N}, \hat{P}_{q} \propto \frac{\alpha_{s} m_{q}}{\sqrt{\hat{s}}}
$$

due to large value of the hard scale $\sqrt{\hat{s}} \sim p_{\perp}$ and small values of $\alpha_{s}$ and $m_{q}$. Here $m_{q}$ stands for the mass of the current quark. Explicit calculation of $s$-quark polarization in gluon fusion process yield $\hat{P}_{s} \leq 4 \%$ [9]. Thus, a straightforward collinear factorization leads to very small $P_{\Lambda}$.

Several modifications of this simple perturbative QCD scheme have been proposed. The factorization formula including higher twist contributions was obtained in [10] and it was recently proposed to consider also twist-3 contributions [11]

$$
E_{a / A}\left(\xi_{1 A}, \xi_{2 A}\right) \otimes q_{b / B}\left(\xi_{B}\right) \otimes \delta D_{c / \Lambda}(z) \otimes d \hat{\sigma}_{a b \rightarrow c d}
$$

as a source for the strange quark polarization [12]. Here the function $E_{a / A}\left(\xi_{1}, \xi_{2}\right)$ is a twist-3 unpolarized distribution, and higher twist partonic correlations could also give additional contributions to fragmentation functions. Since there is no information on higher twist contributions, additional assumptions and models are necessary. Moreover it is not clear what the dynamical origin is of strange quark polarization that arising through the unpolarized quark-gluon correlator $E_{a / A}\left(\xi_{1}, \xi_{2}\right)$. The predicted decreasing dependence $P_{\Lambda} \sim 1 / p_{\perp}$ at high $p_{\perp}$ still does not correspond to the experimental trends.

Note that the situation now appeares to be complicated even for the standard leading twist parton distributions which, are not simply related to the structure 
functions measured in deep-inelastic scattering as it was commonly accepted until now [13]. The crucial role here belongs to the final-state interactions, which could provide a phase difference and lead to non-zero single-spin asymmetries in deepinelastic scattering [14].

Another modification of the collinear factorization takes into account a role of $k_{\perp}$-effects. By analogy with Collins' suggestion for the fragmentation of a transversely polarized quark! it was proposed to consider so called polarizing fragmentation functions (as it was demonstrated recently by Collins [16], the $k_{\perp^{-}}$ effects in distribution functions [17] proposed by Sivers, are, in fact, compatible with $T$-invariance, but those are not relevant for the case of the hyperon polarization in the processes where initial hadrons are unpolarized) for an unpolarized quark with momentum $\mathbf{p}_{\mathbf{q}}$ to fragment into a spin $1 / 2$ hadron $h$ with momentum $\mathbf{p}_{\mathbf{h}}=z \mathbf{p}_{\mathbf{q}}+\mathbf{k}_{\perp}$ and polarization vector $\mathbf{P}_{h}$ [18]:

$$
D_{h \uparrow / q}\left(z, \mathbf{k}_{\perp}\right)=\frac{1}{2} D_{h / q}\left(z, k_{\perp}\right)+\frac{1}{2} \Delta^{N} D_{h \uparrow / q}\left(z, k_{\perp}\right) \frac{\hat{\mathbf{P}}_{h} \cdot\left(\mathbf{p}_{\mathbf{q}} \times \mathbf{k}_{\perp}\right)}{\left|\mathbf{p}_{\mathbf{q}} \times \mathbf{k}_{\perp}\right|}
$$

in the framework of the generalized factorization scheme - with the inclusion of intrinsic transverse motion - along with pQCD dynamics. Thus, in this approach, the source of $\Lambda$ polarization was shifted into the polarizing fragmentation function. Some features of this scheme are:

- a falling $\sim\left\langle k_{\perp}\right\rangle / p_{\perp}$ dependence of the polarization at large $p_{\perp}$,

- still need a dynamical model for polarizing fragmentation functions,

- it is not compatible with the $e^{+} e^{-}$- data at LEP, where no significant transverse polarization of the $\Lambda$ was found [19].

The overall conclusion is that the dynamics of $\Lambda$ polarization as well as of other single spin asymmetries within $p Q C D$ (leading twist collinear factorization or its modifications) is far from being settled. The essential point here is the assumption that at short distances the vacuum is perturbative. The study of the $p_{\perp}-$ dependence of the one-spin asymmetries can be used as a way to reveal the transition from the non-perturbative phase $(P \neq 0)$ to the perturbative phase $(P=0)$ of QCD. The very existence of such transition can not be taken for granted since the vacuum, even at short distances, could be filled with the fluctuations of gluon or quark fields. The measurements of the one-spin transverse asymmetries and polarization will be an important probe of the chiral structure of the effective QCD Lagrangian.

\footnotetext{
${ }^{1}$ It should be noted, that the contributions of instantons to the quark fragmentation could provide necessary helicity flip, but it also leads to vanishingly small single spin asymmetry at large $p_{\perp}$ starting with $p_{\perp} \simeq 2.5 \mathrm{GeV} / \mathrm{c}[15]$.
} 
At the same time we can note that polarization effects as well as some other recent experimental data demonstrate that hadron interactions have a significant degree of coherence. The persistence of elastic scattering at high energies and significant polarization effects at large angles confirm this [20]. It means that the polarization dynamics has its roots hidden in the genuine non-perturbative sector of QCD. Several models exploiting confinement and chiral symmetry breaking have been proposed. The best known are the model based on the classical string picture (Lund model) where the polarization of a strange quark is the result of angular momentum conservation and the model based on the polarization of strange quark due to the Thomas precession. The main feature of the both models is that these take into account for the consequences of quark confinement. The most recent discussion of the successes and failures of these models can be found in [6].

It is worth noting that the chiral $S U(3)_{L} \times S U(3)_{R}$ group of the QCD Lagrangian is not observed in the hadron spectrum. In the real world this group is broken down to $S U(3)_{V}$ with the appearance of the $N_{f}^{2}-1=8$ Goldstone bosons ( $\pi, \mathrm{K}$ and $\eta$ ). Chirality, being a symmetry of QCD Lagrangian, is broken (hidden) by the vacuum state

$$
\langle 0|\bar{\psi} \psi| 0\rangle=\left\langle 0\left|\bar{\psi}_{L} \psi_{R}+\bar{\psi}_{R} \psi_{L}\right| 0\right\rangle
$$

The scale of the spontaneous chiral symmetry breaking is $\Lambda_{\chi} \simeq 4 \pi f_{\pi} \simeq 1 \mathrm{GeV}$, where $f_{\pi}$ is the pion decay constant. Chiral symmetry breaking generates quark masses:

$$
m_{U}=m_{u}-2 g_{4}\langle 0|\bar{u} u| 0\rangle-2 g_{6}\langle 0|\bar{d} d| 0\rangle\langle 0|\bar{s} s| 0\rangle .
$$

Massive quarks are quasiparticles (current quarks surrounded by cloud of quarkantiquark pairs of different flavors). It is worth to stress that in addition to $u$ and $d$ quarks the constituent quarks ( $U$, for example) contain pairs of strange quarks and the ratio of the scalar density matrix elements

$$
y=\langle U|\bar{s} s| U\rangle /\langle U|\bar{u} u+\bar{d} d+\bar{s} s| U\rangle
$$

is estimated as $y=0.1-0.5$. The spin of the constituent quark $J_{U}$ is

$$
\begin{gathered}
J_{U}=1 / 2=S_{u_{v}}+S_{\{\bar{q} q\}}+L_{\{\bar{q} q\}}=1 / 2+S_{\{\bar{q} q\}}+L_{\{\bar{q} q\}} . \\
(\Delta \Sigma)_{U}=1 / 2+S_{\{\bar{q} q\}}, \quad(\Delta \Sigma)_{p}=(\Delta U+\Delta D)(\Delta \Sigma)_{U}, \quad(\Delta \Sigma)_{p} \simeq 0.2,
\end{gathered}
$$

leading to $L_{\{\bar{q} q\}} \simeq 0.4$ Accounting for the axial anomaly in the framework of chiral quark models results in the compensation of the valence quark helicity by helicities of quarks from the cloud in the structure of constituent quark. The specific 
nonperturbative mechanism of such compensation may be different in different models.

Orbital angular momentum, i.e. orbital motion of quark matter inside constituent quark, could generate the observed asymmetries in inclusive production on a polarized target at moderate and high transverse momenta [21]. The main points of the mechanism proposed in [21] are:

- the asymmetry in the pion production reflects the internal spin structure of the constituent quarks, i.e. it arises from the orbital angular momentum of the current quarks inside the constituent quark structure;

- the sign of the asymmetry and its value are proportional to the polarization of the constituent quark inside the polarized nucleon.

Another model [22] which relates the asymmetries to the orbital motion considers these a result of the orbiting valence quarks. It is not yet clear if it is possible to reconcile the orbital motion of valence quarks with the spectroscopy data.

The generic behavior of the different asymmetries in inclusive meson and hyperon production

$$
P, A_{N}\left(s, x, p_{\perp}\right)=\frac{\sin \left[\mathcal{P}_{\tilde{Q}}(x)\left\langle L_{\{\bar{q} q\}}\right\rangle\right] W_{+}^{h}\left(s, x, p_{\perp}\right)}{W_{+}^{s}\left(s, x, p_{\perp}\right)+W_{+}^{h}\left(s, x, p_{\perp}\right)},
$$

(where the functions $W_{+}^{s, h}$ are determined by the interactions at large and small distances) was predicted [21] to have a characteristic $p_{\perp}$-dependence, in particular: vanishing asymmetry for $p_{\perp}<\Lambda_{\chi}$, an increase in the region of $p_{\perp} \simeq \Lambda_{\chi}$, and a $p_{\perp}$-independent (flat) asymmetry for $p_{\perp}>\Lambda_{\chi}$ (Fig. 1).

The parameter $\Lambda_{\chi} \simeq 1 \mathrm{GeV} / \mathrm{c}$ is determined by the scale of spontaneous chiral symmetry breaking. This behavior of the asymmetry follows from the fact that the constituent quarks themselves have a slow (if at all) orbital motion and are in an $S$-state. Interactions with $p_{\perp}>\Lambda_{\chi}$ resolve the internal structure of constituent quark and "feel" the presence of internal orbital momenta inside this constituent quark. The polarization of $\Lambda$-hyperons in the model [7] is result of the polarization the constituent quark due to multiple scattering in the effective field. It means that $s$-quark in the structure of constituent quark will be polarized too, since in the model the spins of quark-antiquark pairs and their angular orbital momenta are exactly compensated:

$$
L_{\{\bar{q} q\}}=-S_{\{\bar{q} q\}} .
$$

It should be noted that DIS data show that strange quarks are negatively polarized in polarized nucleon, $\Delta s \simeq-0.1$. Also elastic $\nu p$-scattering data provide a negative value $\Delta s=-0.15 \pm 0.08$ [23]. The presence and polarization of strange 

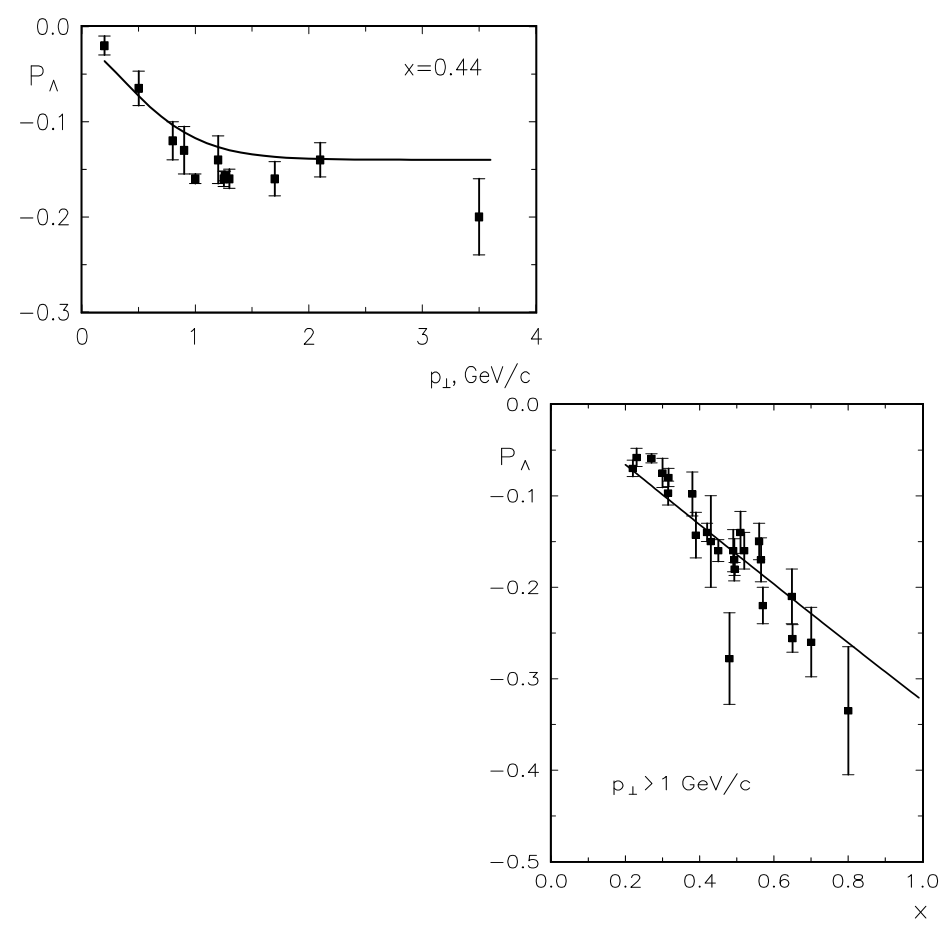

Figure 1: Transverse momentum and Feynman $x$ dependence of $P_{\Lambda}$.

quarks inside a hadron should also give an experimental signal in hadronic reactions.

The parent constituent quark and the strange quark have opposite polarization. The size of the constituent quark is determined by the scale of the chiral symmetry breaking, i. e. $R_{Q} \simeq 1 / \Lambda_{\chi}$. The polarization is significant when the interaction resolves the internal structure of the constituent quark: $r<R_{Q}$ i. e. for $p_{\perp}>\Lambda_{\chi} \simeq 1 \mathrm{GeV} / \mathrm{c}$. It is useful to note here that current quarks appear in the nonperturbative vacuum and become quasiparticles due to the nonperturbative "dressing up" with a cloud of $\bar{q} q$-pairs. The mechanism of this process could be associated with the strong coupling existing in the pseudoscalar channel.

Thus, this model $[\nabla]$ predicts a similarity of the $p_{\perp}$-dependencies for the different spin observables: increase up to $p_{\perp}=\Lambda_{\chi}$ and flat dependence at $p_{\perp}>\Lambda_{\chi}$.

The transition to the partonic picture in this model is described by the introduction of a momentum cutoff $\Lambda=\Lambda_{\chi} \simeq 1 \mathrm{GeV} / \mathrm{c}$, which corresponds to the scale of spontaneous chiral symmetry breaking. We shall see that at higher $p_{\perp}$ the constituent quark to be a cluster of partons, which however should preserve their orbital momenta, i.e. the orbital angular momentum will be retained and the partons in the cluster are correlated. Note, that the short-distance interaction in this approach observes a coherent rotation of correlated $\bar{q} q$-pairs inside the 
constituent quark and not a gas of the free partons. The nonzero internal orbital momenta of partons in the constituent quark means that there are significant multiparton correlations. Indeed, a high locality of strange sea in the nucleon was found experimentally by the CCFR collaboration at FNAL. This locality serves as a measure of the local proximity of strange quark and antiquark in momentum and coordinate space. It was shown [24] that the CCFR data indicate that the strange quark and antiquark have very similar distributions in momentum and coordinate space.

\section{Experimental prospects}

Experimentally observed persistence and constancy of $\Lambda$-hyperon polarization means [7] that chiral symmetry is not restored in the region of energy and values of $p_{\perp}$ where experimental measurements were performed. Otherwise we would not have any constituent quarks and should expect a vanishing polarization of $\Lambda$ [7]. It is interesting to perform $\Lambda$-polarization measurements at RHIC and the LHC. It would allow to make a direct check of perturbative QCD and allow to make a cross-check of the QCD background estimations based on perturbative calculations for the LHC. It is an important problem which still awaits its solution.

Experimentally measurable effects of $10 \%$ or higher are expected at c.m.s. energy $\sqrt{s}=500 \mathrm{GeV}$ for values of pseudorapidity $|\eta| \geq 2.3$, and for energies 2 $\mathrm{TeV}$ and $10 \mathrm{TeV}$ at $|\eta| \geq 3.7$ and $|\eta| \geq 5$ respectively.

The use of a polarized beam will allow to measure the two-spin correlations in hyperon production at $\mathrm{TeV}$ energies at the $\mathrm{LHC}$, and to reveal the underlying mechanism. Moreover, measurements of the transverse and longitudinal asymmetries provide information on production mechanism and on hadron spin structure. It seems very promising to study reactions with weakly decaying baryons in the final state such as

$$
p_{\uparrow, \rightarrow}+p \rightarrow \Lambda_{\uparrow, \rightarrow}+X
$$

and to measure the parameters $D_{N N}$ and $D_{L L}$. These asymmetries are calculable in the framework of perturbative QCD [25, 26]. The data on $D_{N N}$ and $D_{L L}$ in the fragmentation region at large $x_{F}$ seem to be interesting also from the point of view of the polarization of the strange sea and strangeness content of the nucleon. The strange sea has a large negative polarization value according to the interpretation of the polarized deep-inelastic scattering results, and it should be revealed in $D_{N N}$ and $D_{L L}$ behavior. The typical behavior of spin parameters can be illustrated by the dependence of asymmetry $A_{N}$ on $p_{\perp}$ (Fig. 2) 


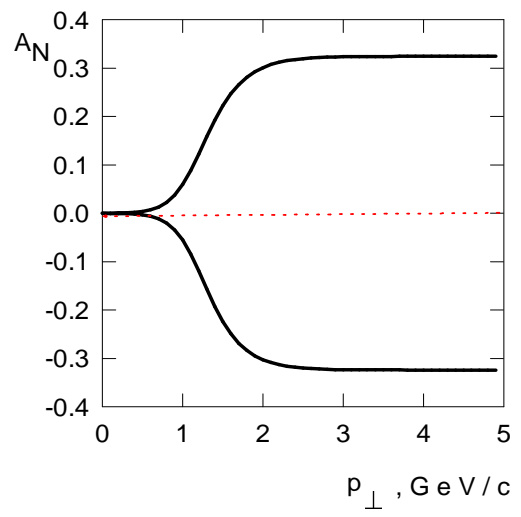

Figure 2: The dependence of $A_{N}$ in charged pion production at $\sqrt{s}=500 \mathrm{GeV}$ and $x=0.5$

The asymmetries have energy independent values about $30 \%$ at $p_{\perp}>\Lambda_{\chi}(\simeq 1-2$ $\mathrm{GeV} / \mathrm{c}$ ) and $x \simeq 0.5$.

Note, that the corresponding asymmetry in neutral pion production has a value about $5-6 \%$ in the same kinematical region.

If two polarized nucleons are available in the initial state then one could measure three-spin parameters such as $(n, n, n, 0)$ and $(l, l, l, 0)$ in the process:

$$
p_{\uparrow, \rightarrow}+p_{\uparrow, \rightarrow}=\Lambda_{\uparrow, \rightarrow}+X
$$

where the polarization of $\Lambda$-hyperon is studied through its decay. Measurements of the three-spin correlation parameters would provide important data for the study of hyperon production dynamics and mechanisms for hyperon polarization. On that basis we expect, as it was already mentioned, similar $p_{\perp}$-dependence for all spin parameters when initial hadrons have no valence strange quarks.

When one of the colliding hadrons has a valence strange quark, the picture will include two mechanisms: the one described above and another one - polarization of valence strange quarks in the effective field. The latter mechanism will provide $\Lambda$ 's polarized in the opposite direction and can explain the positive large polarization $P_{\Lambda}$ in $K^{-} p$-interactions.

As it was claimed in [27] on the basis of DIS nonperturbative analysis, that the orbital angular momentum increases with virtuality $Q^{2}$. Thus, in the framework of the model studied and according to the relation (5) it would lead to increasing polarization of strange quark. It means, in particular, that the transverse polarization $P_{\Lambda}\left(Q^{2}\right)$ in the current fragmentation region of the semi-inclusive process

$$
l+p \rightarrow l+\Lambda_{\uparrow}+X
$$


will also increase with virtuality, provided the competing mechanism of the polarization of the valence strange quark from the $\varphi$-meson gives a small contribution. Regardless of these particular predictions, it seems that the experimental studies of polarization dependence on virtuality could shed new light on this problem.

\section{Quark-gluon plasma detection}

On the base of the model in [7] one expects zero polarization in the region where QGP has formed, since chiral symmetry is restored and there is no room for quasiparticles such as constituent quarks. The absence or strong diminishing of transverse hyperon polarization can be used therefore as a signal of QGP formation in heavy-ion collisions. This prediction should also be valid for the models based on confinement, e.g. the Lund and Thomas precession model. In particular, the polarization of $\Lambda$ in heavy-ion collisions in the model based on the Thomas precession was described in [28] where nuclear effects were discussed as well. However, we do not expect a strong diminishing of the $\Lambda$-polarization due to the nuclear effects: the available data show a weak $A$-dependence and are not sensitive to the type of the target. Thus, we could use a vanishing polarization of $\Lambda$-hyperons in heavy ion collisions as a sole result of QGP formation provided the corresponding observable is non-zero in proton-proton collisions. The prediction based on this observation would be a decreasing behavior of polarization of $\Lambda$ with the impact parameter in heavy-ion collisions in the region of energies and densities where QGP was produced:

$$
P_{\Lambda}(b) \rightarrow 0 \quad \text { at } \quad b \rightarrow 0,
$$

since the overlap is maximal at $b=0$. The value of the impact parameter can be controlled by the centrality in heavy-ion collisions. The experimental program should therefore include measurements of $\Lambda$-polarization in $p p$-interactions first, and then if a significant polarization would be measured, the corresponding measurements could be a useful tool for the QGP detection. Such measurements seem to be experimentally feasible at RHIC and LHC provided it is supplemented with forward detectors.

\section{Conclusion}

In conclusion we would like to emphasize the main points of our discussion:

- a universal $p_{\perp}$-dependence for all spin parameters in $\Lambda$-hyperon production which reflects a finite size for constituent quarks is predicted: all spin pa- 
rameters in the collisions of hadrons without valence strange quarks demonstrate an increase in absolute value up to $p_{\perp} \simeq 1 \mathrm{GeV} / \mathrm{c}$ and then become flat.

- three-spin correlation parameters - the new observables which can be measured in hyperon production with polarized beams - can provide new insight into the mechanism of hyperon polarization

- hyperon polarization should vanish in heavy-ion collisions when quarkgluon plasma is formed, its decrease with centrality can be considered as a gold-plated signature of QGP formation.

\section{Acknowledgements}

We are grateful to A. De Roeck for the useful suggestions, comments and careful reading of the manuscript, V. Petrov and P. Schlein for the interesting discussions.

\section{References}

[1] G. Bunce, N. Saito, J. Soffer, W. Vogelsang, Ann. Rev. Nucl. Part. Sci. 50, 525, (2000).

[2] G. Bunce at al., Phys. Rev. Lett. 36 , (1976) 1113;

For a history of the hyperon polarization discovery, see: T. Devlin, in SPIN 94, Proceedings of the 11th International Symposium on High-energy Spin Physics and the 8th International Symposium on Polarization Phenomena in Nuclear Physics, Bloomington, Indiana, 15-22 September 1994, AIP Conf. Proc. Woobury, New York, 1995, eds. K. Heller and S. Smith, p. 354.

[3] L. Pondrom, Phys. Rep. 122, (1985) 57;

K. Heller, in Proceedings of the 7th International Symposium on High-Energy Spin Physics, Protvino, Russia, 1987, p. 81;

J. Duryea et al., Phys. Rev. Lett. 67 , (1991) 1193.

A. Morelos et al., Phys. Rev. Lett. 71 , (1993) 2172;

K. A. Johns et al., in SPIN 94, Proceedings of the 11th International Symposium on High-energy Spin Physics and the 8th International Symposium on Polarization Phenomena in Nuclear Physics, Bloomington, Indiana, 15-22 September 1994, AIP Conf. Proc. Woobury, New York, 1995, eds. K. Heller and S. Smith, p. 417;

L. Pondrom, ibid., p. 365;

A. D. Panagiotou, Int. J. Mod. Phys A 5, 1197 (1990).

[4] A. Bravar, in SPIN 98 Proceedings of the 13th International Symposium on HighEnergy Spin Physics, Protvino, Russia, 8-12 September 1998, N. E. Tyurin, V. L. 
Solovianov, S. M. Troshin, and A. G. Ufimtsev, eds. (World Scientific, Singapore, 1999) p. 167.

[5] T. Henkes et al. (R608 Collaboration ), Phys. Lett. B 283, 155 (1992)

[6] J. Felix, Mod. Phys. Lett. A14, 827 (1999);

J. Soffer, Invited talk at Hyperon 99 Hyperon Physics Symposium, Batavia, Illinois, 27-29 Sep 1999;

Liang Zuo-tang and C. Boros, Int. J. Mod. Phys. A 15, 927 (2000).

[7] S. M. Troshin and N. E. Tyurin, Phys. Rev. D55, 1265 (1997).

[8] G. L. Kane, J. Pumplin, and W. Repko, Phys. Rev. Lett. 41, 1689 (1978).

[9] W. G. D. Dharmaratna and G. R. Goldstein, Phys. Rev. D 53, 1073 (1996)

[10] J. Qiu and G. Sterman, Phys. Rev. D 59, 014004 (1999).

[11] A. V. Efremov and O. V. Teryaev, Sov. J. Nucl. Phys. 36, 140 (1982).

[12] Y. Kanazawa and Y. Koike, Phys. Rev. D, 64, 034019 (2001).

[13] S. J. Brodsky, P. Hoyer, N. Marchal, S. Peigné, and F. Sannino, SLAC-PUB-8818, hep-ph/0104291; S. Peigné and F. Sannino, hep-ph/0112080.

[14] S. J. Brodsky, D. S. Hwang and I. Schmidt, hep-ph/0201296.

[15] N. I. Kochelev, JETP Lett. 72, 481 (2000).

[16] J. C. Collins, hep-ph/0204004.

[17] D. W. Sivers, Phys. Rev. D 41, 83 (1990).

[18] M. Anselmino, D. Boer, U. D’Alesio, and F. Murgia, Phys. Rev. D. 63, 054029 (2001).

[19] D. Buskulic et al. (ALEPH Collaboration), Phys. Lett. B 374, 319 (1996).

[20] S.M. Troshin and N.E. Tyurin, Phys. Lett. B 144, 260, 1984.

[21] S. M. Troshin and N. E. Tyurin, Phys. Rev. D52, 3862 (1995), ibid. D54, 838 (1996).

[22] C. Boros and Liang Zuo-tang, Phys. Rev. D 53, R2279 (1996);

Liang Zuo-tang and C. Boros, Phys. Rev. Lett. 79, 3608 (1997).

[23] L. A. Ahrens et al. Phys. Rev. D35 , 785 (1987); see for interpretation of the data S.

D. Bass and A. W. Thomas, J. Phys. G 19 , 925 (1993).

[24] X. Ji and J. Tang, Phys. Lett. B 362, 182 (1995).

[25] N. S. Craigie, K. Hidaka, M. Jacob and F. M. Renard, Phys. Rep. 99, 69 (1983). 
[26] C. Bourelly, J. Soffer, F. M. Renard and P. Taxil, Phys. Rep. 177, 319 (1989).

[27] S.M. Troshin and N.E. Tyurin, hep-ph/0109047.

[28] N. Angert et al., in Proceedings of the Conference on Quark Matter Formation and Heavy Ion Collisions, eds. M. Jacob and H. Satz, World Scientific, Singapore, 1982;

A. D. Panagiotou, Phys. Rev. C 33, 1999 (1986);

A. Ayala, E. Cuautle, G. Herrera, and L . Montano, nucl-th/0110027. 